\title{
Optimisation fonctionnelle de production par rodage des surfaces des fûts de moteur
}

\author{
Leila Sabri ${ }^{1, a}$, Sabeur Mezghani ${ }^{2}$ et Mohamed El Mansori ${ }^{2}$ \\ 1 RENAULT S.A.S., Direction de la Mécanique/Direction de l'Ingénierie Process, 67 rue des Bons Raisins, \\ 92508 Rueil Malmaison, France \\ 2 Arts et Métiers ParisTech, LMPF-EA 4106, rue Saint-Dominique, BP 508, 51006 Châlons-en-Champagne Cedex, France
}

Reçu le 15 octobre 2009, accepté le 12 juillet 2010

\begin{abstract}
Résumé - Face au réchauffement climatique lié à l'effet de serre, les constructeurs automobiles poursuivent leurs efforts dans la réduction des émissions de $\mathrm{CO}_{2}$. Le ticket d'entrée pour répondre à ces contraintes, tout en réduisant les coûts d'investissement, est d'optimiser les technologies de finition des fûts de carter cylindres des moteurs. Toutefois, l'acceptation des surfaces issues de ces technologies de finition, notamment le rodage, est pilotée par un contrôle visuel associé à des critères empiriques d'état et aspect de surface. Le but du présent travail est de proposer une méthodologie objective de caractérisation de la qualité de surface, basée sur une analyse multi-échelle de la texture. Différents critères de topographie et d'aspect de surface ont été définis. Ils ont été appliqués pour l'étude de l'impact de la vitesse d'expansion des rodoirs sur les variations de la qualité de surface obtenue. Des essais instrumentés ont été menés, afin de suivre l'évolution des paramètres physiques relatifs au rodage, tels que les énergies spécifiques requises à chacune des configurations. Cette étude démontre le potentiel de la méthode développée à définir des corrélations objectives entre les paramètres process et la qualité de surface après rodage.
\end{abstract}

Mots clés : Rodage / abrasion / caractérisation de surface / rugosité / décomposition multi-échelle

\begin{abstract}
Functionnal optimization of production by honing engine cylinder liner. Nowadays, the improvement of the combustion engines efficiency in the automotive field is the main key towards the reduction of greenhouse gas emission. The improvement demanded with lower productive cost can be either in the method of manufacturing of crankcase cylinder liner. However, the performance quantification of these processes, especially the honing process, is tightly related to a visual control with some empirical roughness criteria. The purpose of this study is to probe an objective methodology for the characterization of honed surfaces based on multi-scale analysis of the surface. Sensible surface texture characteristics were derived and were used to investigate the influence of the abrasive stones and the expansion velocity in honing. Based on a series of instrumented honing tests, the method shows its powerful ability to detect objective correlations between the honing process parameters and the quality of honed surfaces.
\end{abstract}

Key words: Honing / abrasion / surface characterization / roughness / multi-scale decomposition

\section{Introduction}

Dans un contexte de durcissement des normes environnementales et des exigences de la clientèle en termes de performances et confort, les constructeurs automobiles ont engagé une course à la performance moteur et aux moyens de répondre à ces demandes. Il a été démontré par ailleurs que le compartiment segment-piston-chemise présente $40 \%$ des pertes par frottement moteur [1]. Aussi, un grand intérêt a été exprimé à l'étude des procédés

\footnotetext{
a Auteur pour correspondance :

leila.sabri@renault.com
}

de finition des surfaces de fûts de carter cylindres. En effet, ces surfaces conditionnent les propriétés fonctionnelles du moteur telle que la consommation d'huile, les phénomènes de blow-by ${ }^{1}$ ou encore l'usure du contact segment/fût ou chemise. La texture interne des cylindres de fûts est généralement obtenue par le procédé de rodage

\footnotetext{
1 Les gaz de blow-by proviennent principalement des fuites au niveau de la segmentation. Ces gaz, une fois dans le carter moteur, se chargent en aérosol d'huile. Les gaz de blow-by sont source de désagréments car ils entraînent la mise en pression du carter et participent à la consommation d'huile.
} 


\section{Nomenclature}

\begin{tabular}{|llr|}
\hline$\theta$ & Angle de rodage & $\left({ }^{\circ}\right)$ \\
$C r$ & Critère de rodage & $(\mu \mathrm{m})$ \\
$C f$ & Critère de fonctionnement & $(\mu \mathrm{m})$ \\
$C l$ & Critère de lubrification & $(\mu \mathrm{m})$ \\
$M C r$ & Critère multi-échelle de rodage & $(\mu \mathrm{m})$ \\
$M C f$ & Critère multi-échelle de fonctionnement & $(\mu \mathrm{m})$ \\
$M C l$ & Critère multi-échelle de lubrification & $(\mu \mathrm{m})$ \\
$S M a$ & Critère multi-échelle de rugosité & $(\mu \mathrm{m})$ \\
$D_{\mathrm{s}}$ & Densité des stries & $\left(\right.$ stries. $\left.\mathrm{mm}^{-2}\right)$ \\
$E_{\mathrm{sp}}$ & Energie spécifique & $(\mathrm{J})$ \\
$P M H$ & Point mort haut & $(\mathrm{mm})$ \\
$P M B$ & Point mort bas & $(\mathrm{mm})$ \\
$P M$ & Point milieu & $(\mathrm{mm})$ \\
$P_{\mathrm{c}}$ & Puissance consommée & $(\mathrm{W})$ \\
$R_{\mathrm{s}}$ & Rapport des stries & $(1)$ \\
$Q_{w}$ & Taux d'enlèvement de matière & $(\mathrm{s})$ \\
$t$ & Temps de rodage & $\left(\mathrm{mm}^{3} \cdot \mathrm{s}^{-1}\right)$ \\
$W$ & Volume de matière enlevée & $\left(\mathrm{mm}^{3}\right)$ \\
\hline
\end{tabular}

avec abrasifs agglomérés. La cinématique du procédé est assurée par la combinaison de trois mouvements (Fig. 1) :

- un mouvement de rotation de la broche avec un moteur mécanique $\left(V_{\mathrm{r}}\right)$;

- un mouvement axial donné par un système pneumatique $\left(V_{\mathrm{a}}\right)$;

- un mouvement d'expansion des pierres de rodage une fois l'outil de rodage dans la pièce à roder $\left(V_{\exp }\right)$.

L'obtention de la qualité du fini de surface des carters nécessite souvent la succession de trois étapes de rodage [2] :

1. rodage ébauche pour l'amélioration de la forme et la définition de la striation initiale;

2. rodage finition pour la réduction de la rugosité et l'amélioration de l'aspect;

3. rodage plateau pour supprimer les pics et obtenir des stries plateau.

Toutefois, ce procédé se heurte à la difficulté liée à l'empirisme du choix des paramètres process relatifs à chaque nouvelle spécification fonctionnelle. Il est à préciser que jusqu'à présent, la mise au point d'une gamme de rodage des chemises s'est fondée sur des bases de données expérimentales adaptées à la nouvelle définition de la surface.

Outre l'aspect mise au point du process, la caractérisation des surfaces est une étape inéluctable dans l'indentification et l'optimisation du procédé de rodage. La méthode de caractérisation retenue doit fournir le maximum d'informations sur la topographie de la surface afin d'extraire la signature la plus représentative possible du process. À l'instar des autres constructeurs automobiles, Renault a adopté sur ses lignes de production une technique d'analyse basée sur un contrôle qui regroupe deux types de critères :

- critères basés sur des paramètres de rugosité;

- critères fondés sur appréciation visuelle de l'aspect de la surface.

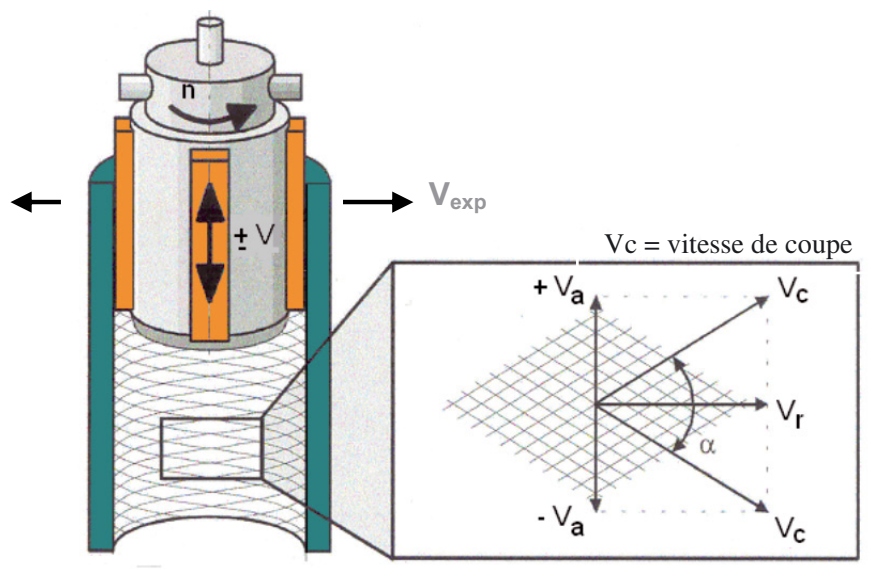

(a)

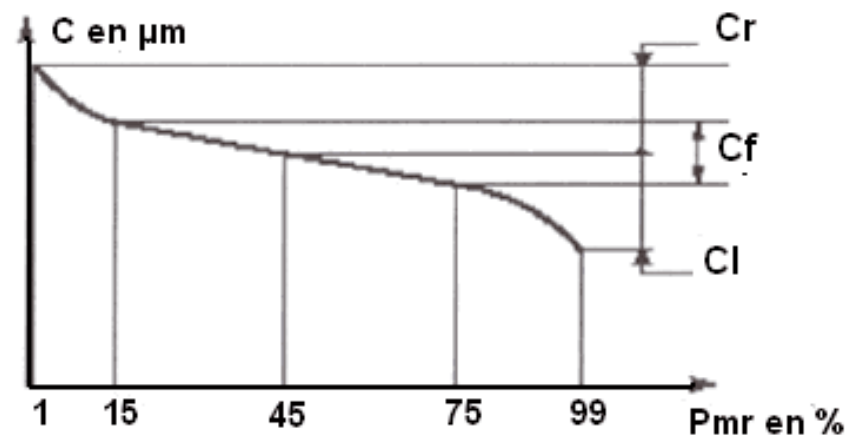

(b)

Fig. 1. (a) Illustration des trois mouvements simultanés au cours d'une opération de rodage. (b) Les paramètres de la norme CNOMO définis à partir de la courbe de portance.

\section{Critères de rugosité}

Les paramètres de rugosité sont calculés à partir de la courbe d'Abbott qui représente le taux des zones portantes à chaque niveau de coupe de la surface [3]. 

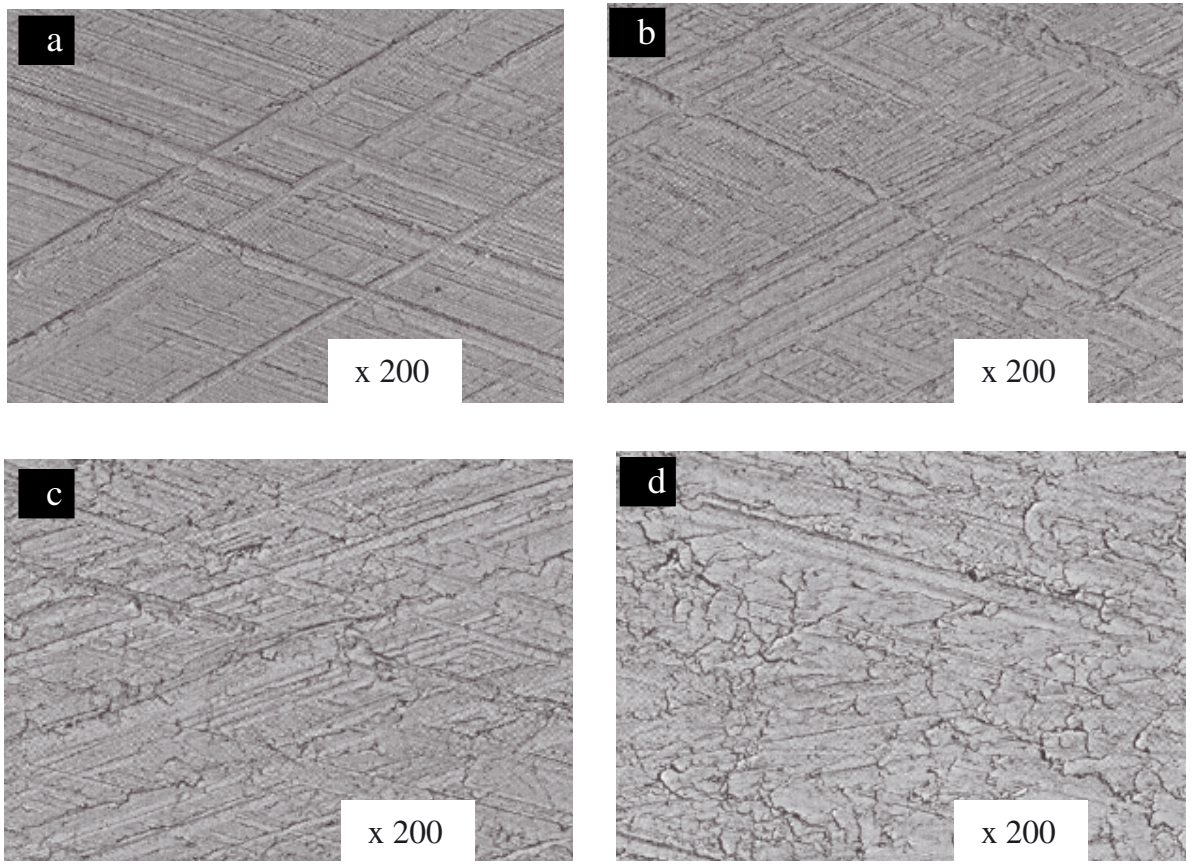

Fig. 2. Les différents types de qualité de surface chemise moteur définis par le constructeur automobile Renault : (a) type 1; (b) type 2 ; (c) type 3 ; (d) type 4 .

Une norme française, appelée norme CNOMO [4], a par ailleurs été introduite par les industriels automobiles français pour mieux renseigner sur les fonctionnalités de la surface. Il s'agit des trois paramètres suivants :

- $C r$ : le critère de rodage, où les pics les plus saillants vont être usés et disparaître pendant les premières heures de fonctionnement. L'absence de ce critère limitera d'autant le temps de rodage d'un moteur. Il est calculé comme étant la différence des hauteurs des plans de coupe correspondant aux taux de portance de $45 \%$ et $1 \%$;

- Cf : le critère de fonctionnement, qui représente la quantité de matière disponible à l'usure. Plus cette quantité est disponible, plus un moteur fonctionnera longtemps. Il est obtenu par la différence des hauteurs des plans de coupe correspondant aux taux de portance de $15 \%$ et $75 \%$;

- Cl : le critère de lubrification, qui détermine les creux disponibles et toujours utiles pour retenir un lubrifiant. L'absence ou la disparition de ces creux entrainera le grippage du moteur. Il est calculé par la différence des hauteurs des plans de coupe correspondant aux taux de portance de $45 \%$ et $99 \%$.

\section{Critères d'aspect}

Les paramètres d'aspect permettent de renseigner sur les propriétés de la morphologie latérale de la surface. Ils peuvent être divisés en deux groupes, caractérisant [5] :

- la texture des stries (comprenant l'angle de rodage, densité des stries, stries résiduelles et la formation des plateaux);
- les irrégularités de surface (incluant toutes les déviations d'une texture idéale comme des graphites lamellaires, trous, défauts, interruption de stries...).

Ces paramètres sont généralement qualifiés par les techniques de microscopie. Une méthode non destructive d'investigation de ces propriétés est l'utilisation des fax film. Elle consiste à presser un film acétate sur les zones à mesurer après avoir appliqué de l'acétone. Après évaporation de l'acétone, le film est enlevé de la surface pour être examiné à l'aide d'un microscope [6]. Un expert définit ensuite une cotation de l'aspect de surface. La procédure Renault définit quatre critères (Fig. 2) permettant de porter un jugement sur la qualité de la surface, à savoir :

- type1 : stries bien croisées et bien marquées;

- type2 : stries croisées et marquées; les languettes sont inférieures à la taille des stries de lubrification;

- type3 : stries croisées et peu marquées; les languettes sont supérieures à la taille des stries de lubrification avec recouvrement partiel des stries;

- type 4 : stries peu marquées recouvertes de languettes et d'un manteau de tôle.

La définition de ces critères reste toutefois tributaire de l'évaluation visuelle et subjective d'un opérateur. De plus, les paramètres statistiques de la topographie de la surface ne permettent pas de renseigner sur les donnée « dynamiques » de la surface dues aux différentes modifications introduites par chacune des étapes du procédé. Afin de pallier à la lourdeur des méthodes actuelles de caractérisation en ligne de production et à leur caractère empirique, une méthodologie objective de caractérisation des surfaces rodées basée sur une analyse multi-échelle de la texture est proposée. Ainsi, nous nous 


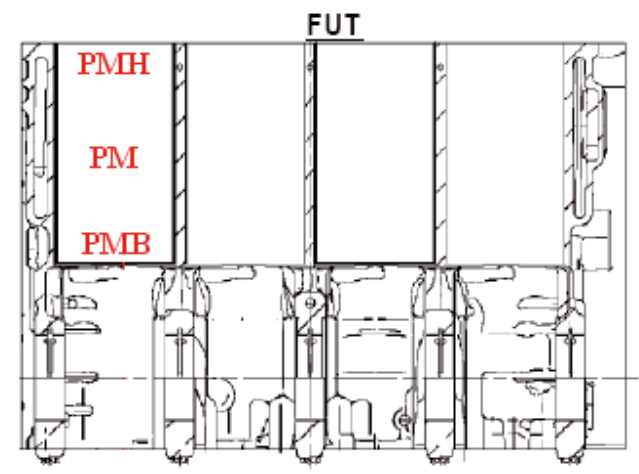

(a)

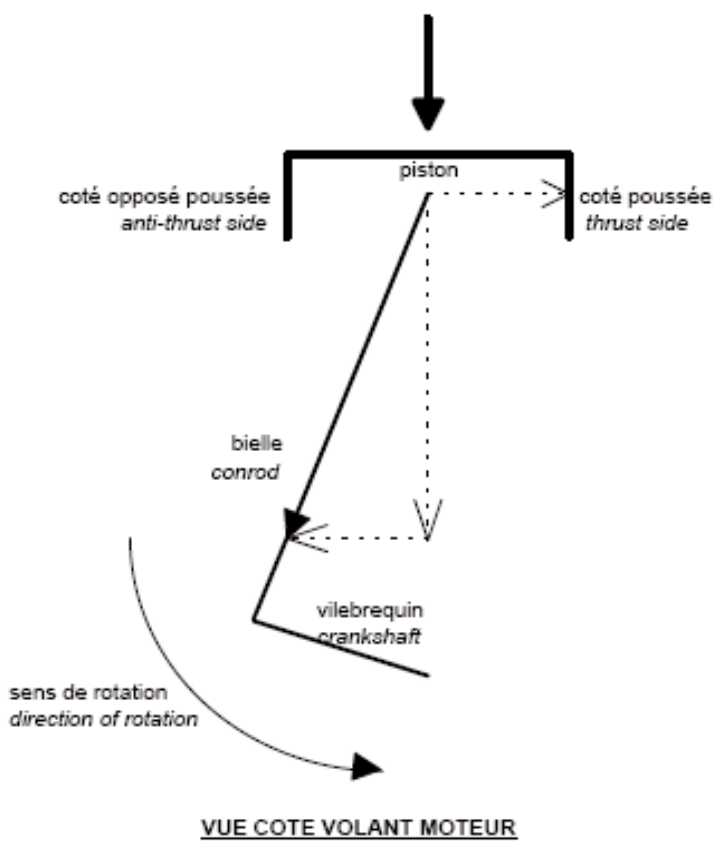

(b)

Fig. 3. (a) Vue de coupe d'un fût de cylindres moteurs précisant les trois zones de mesures PMH (point mort haut), PM (point milieu) et PMB (point mort bas). (b) Repérage du côté poussée.

proposons dans ce travail de décrire le principe de cette méthodologie et de montrer les intérêts de son application à la compréhension ainsi qu'au suivi du procédé de rodage.

\section{Mise en œuvre d'une méthode d'acquisition d'image 3D des surfaces rodées par réplication}

Au lieu de procéder à des mesures profilomètriques associées à des visualisations microscopiques et afin de pallier aux handicaps de ces méthodes sur des surfaces anisotropes (surfaces rodées), l'utilisation de la mesure 3D permet de mettre en place une méthodologie d'analyses des surfaces basée sur le traitement d'une seule mesure. Celle-ci renseignera simultanément sur les caractéristiques topographiques et sur l'aspect de la surface rodée.

Conformément aux exigences industrielles, le moyen de définition de ces paramètres doit être non-destructif. Pour ce faire, nous avons proposé d'utiliser des empreintes par SILFLO composé d'un produit pâteux, d'un catalyseur et d'un adoucisseur. Le mélange est, en effet, étalé sur la surface usinée de la chemise au niveau du point milieu du côté poussée (voir Fig. 3). L'empreinte obtenue sur la réplique représente alors la topographie inversée de la surface de la chemise. Le relevé topographique de la surface chemise moteur est réalisé sur la réplique avec un interféromètre optique 3D à lumière blanche (WYKO $3300 \mathrm{NT}$ ). La surface totale obtenue est le résultat d'un recollement de 9 images (Stitching) en adoptant un taux de recouvrement de $50 \%$. Ce taux de recouvrement important permet de minimiser les défauts de recollage d'images au minimum. L'image topographique obtenue possède les caractéristiques suivantes : elle a une dimension de $1,9 \mathrm{~mm} \times 2,5 \mathrm{~mm}$ correspondant à 640 points dans le sens de la génératrice (sens de déplacement du piston) et de 480 points dans la seconde direction avec un pas de $3,88 \mu \mathrm{m}$.

\subsection{Procédure de traitement des données topographiques 3D}

La phase de traitement des mesures brutes, réalisées sur les répliques, comprend trois étapes :

1. la détermination des altitudes des points non mesurés par interpolation; les paramètres de mesure adoptés ont été choisis de façon à avoir un nombre total de points non mesurés inférieur à $5 \%$;

2. l'inversion de l'image topographique de l'empreinte mesurée sur la réplique; cette inversion permet de retrouver la topographie de la surface chemise;

3. l'extraction de la forme de la surface. Elle consiste à enlever la forme cylindrique de la surface. Cette forme correspond aux composantes basses fréquences et elle constitue un élément non important pour la définition des paramètres d'état de surface. Cette séparation de la forme est réalisée en utilisant l'approximation des moindre carrée basée sur les fonctions Spline cubique. 


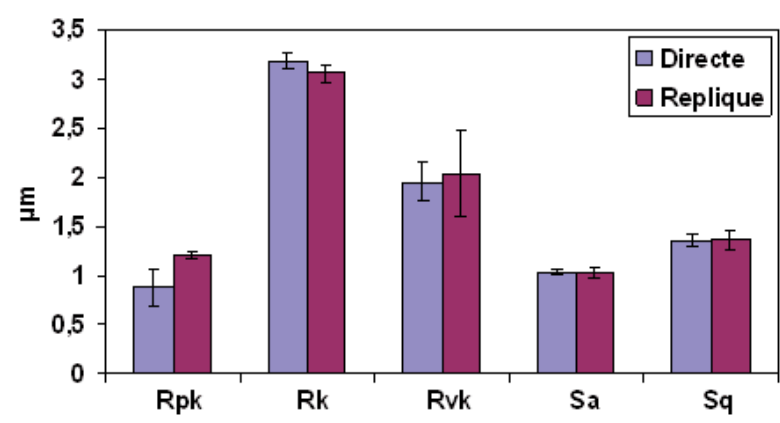

Fig. 4. Comparaison entre les paramètres d'état de surface obtenus par une mesure 3D directe de la surface rodée et celle issue d'une réplique.

\section{2 Étude de la reproductibilité de la méthode}

Afin de valider cette technique de mesure non destructive, on a déterminé l'erreur engendrée entre une mesure directe sur la surface et celle réalisée sur la réplique. Quatre répliques ont alors été réalisées sur la même pièce. Cinq mesures sont effectuées sur différents endroits de la surface et sur chacune des répliques. Les résultats sont reportés sur la figure 4. Compte tenu de ces résultats, il n'existe pas un écart significatif entre la mesure par réplique et la mesure directe sur la surface du fût.

Ainsi, cette méthodologie de mesure a été adoptée pour définir les caractéristiques de la surface. De nouveaux paramètres sont ainsi développés pour mieux caractériser la surface à partir d'une analyse multi-échelle de la topographie et d'une quantification des propriétés d'aspect de la surface avec la transformée de Radon.

\section{Carctérisation multi-échelle de la topographie}

Afin de pallier à la limitation des méthodes d'analyse supposant la stationnarité de la surface, l'approche multi-échelle vient peaufiner l'analyse des caractéristiques de la surface dépendant de différentes échelles. Contrairement aux autres méthodes, elle présente la particularité d'offrir une résolution flexible de la surface avec une description « temps-fréquence » adaptative aux changements de la surface. La fenêtre d'analyse est alors de « petite largeur » pour faire du zoom-in sur les hautes fréquences (petites échelles) et être «plus large » afin de faire du zoom-out sur les petites fréquences (grandes échelles). Ainsi, les échelles deviennent des représentations de l'espace de fréquence permettant d'extraire des données spécifiques de la surface. Cette description est assurée par une décomposition multi-échelle par ondelettes de la surface depuis les échelles d'ondulation jusqu'aux échelles de micro-rugosité.

\subsection{Principe de la décomposition par ondelettes}

Les transformées en ondelettes sont nées au milieu des années quatre-vingts pour répondre aux problèmes de résolution temps-fréquence des méthodes basées sur la transformée de Fourier. En effet, les signaux nonstationnaires nécessitent une analyse dont la résolution fréquentielle (respectivement spatiale) varie avec la localisation spatiale (respectivement fréquentielle). C'est pour permettre cette flexibilité que les ondelettes, un concept d'analyse «multi-résolution » ou « multi-échelle », ont vu le jour. En se basant sur des résultats bibliographiques [7] et compte tenu de la forme des profils des surfaces rodées, nous avons choisi de travailler avec des ondelettes de type chapeau mexicain définies par la formule suivante [8] :

$$
\psi(r)=\left(2-|r|^{2}\right) \mathrm{e}^{-|r|^{2} / 2}
$$

avec $r=(x, y)$.

Cette relation permet d'écrire la transformée en ondelettes 2D sous la forme d'un produit de convolution de la surface avec une base d'ondelettes générée par dilatation et translation :

$$
W_{\psi}^{f}(b, a)=f(x, y) \otimes \psi_{a, b}^{*}(x, y)
$$

avec $b$ paramètre de translation en espace, $a$ paramètre de dilatation relatif à la fréquence et $f(x, y)$ la surface étudiée

La famille d'ondelette peut alors être obtenue en « jouant à l'accordéon» avec l'ondelette analysante définie par l'équation (1). En effet, les ondelettes analysantes sont générées à partir d'une seule ondelette mère. Les coefficients d'ondelettes pour chaque couple de point $(a, b)$ par translation et dilatation de l'ondelette.

\subsection{Définition des paramètres multi-échelles de rugosité}

De nouveaux paramètres multi-échelles de rugosité ont été introduits (Fig. 5).

Ces paramètres sont représentés sous forme de spectres. Chaque valeur dans le spectre définit la valeur du paramètre fonctionnel de rugosité à une échelle donnée et calculé à partir de la courbe d'Abott. Il s'agira alors du paramètre SMa défini comme étant le critère multi-échelle de rugosité arithmétique.

\section{Caractérisation de l'aspect des surfaces rodées}

Pour la caractérisation de l'aspect, il s'agira de définir de nouveaux critères permettant de passer d'une qualification subjective de la surface rodée via la méthode du fax film à une quantification objective de la surface. Pour cela, de nouveaux critères ont été développés en se basant sur la transformée de Radon.

\subsection{Principe de la méthode}

La transformée de Radon permet de transformer des structures linéaires, en l'occurrence les stries de rodage, 


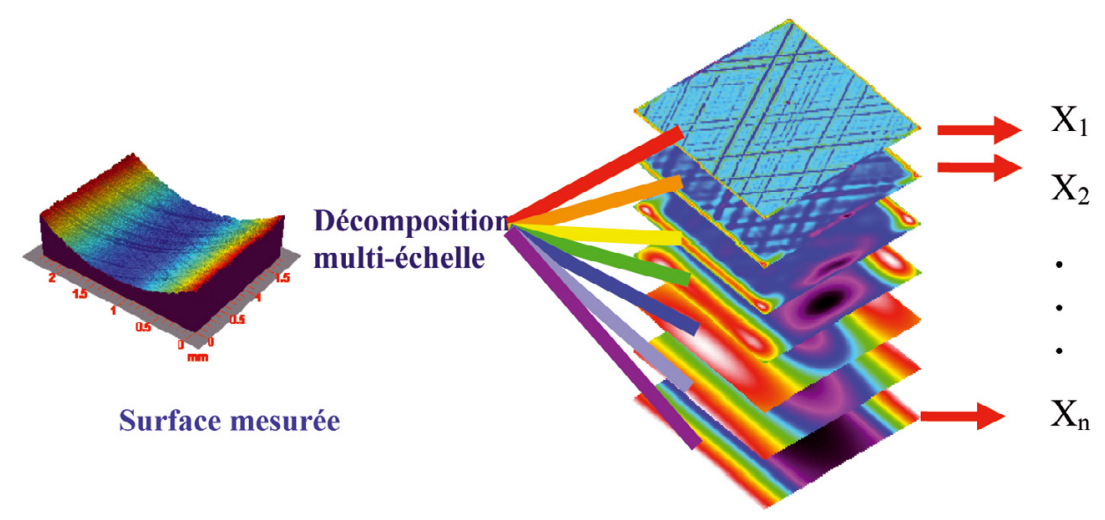

$\mathrm{X}$ : paramètre d'état de surface $(\mathrm{Sa}, \mathrm{Cr}, \mathrm{Cf}, \mathrm{Cl})$

$1 \ldots \mathrm{n}:$ indice d'échelle

Fig. 5. Principe de la décomposition multi-échelle d'une surface de fût de moteur et son spectre multi-échelle de paramètre d'état de surface MX.
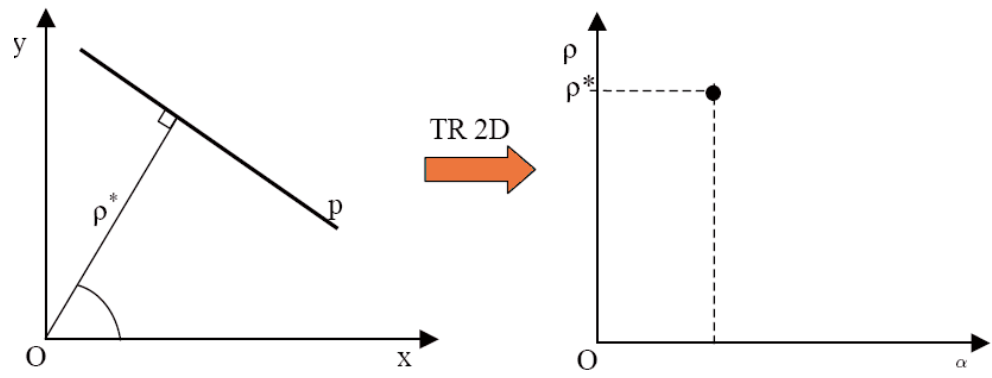

Fig. 6. Principe de la transformée de Radon.

en points singuliers (Fig. 6). Ceci facilitera bien sûr leur quantification et leur classification. Ainsi, la transformée de Radon d'un couple de coordonnées $(\rho, \alpha)$ se formule selon l'expression suivante :

$$
\operatorname{TR}(\rho, \alpha)=\int_{-\infty}^{\infty} \int_{-\infty}^{\infty} f(x, y) \delta(\rho-x \cos \alpha-y \sin \alpha) \mathrm{d} x \mathrm{~d} y
$$

avec $\delta$ la fonction Dirac.

\subsection{Définition des paramètres d'aspect}

La transformée de Radon a été utilisée pour calculer différents paramètres de la texture des surfaces rodées. Nous détaillerons en application dans cette étude les deux paramètres caractérisant l'angle de rodage et la densité des stries.

\subsubsection{Angle de rodage $\ll \theta »$}

L'angle de rodage $« \theta »$ est obtenu par la combinaison du mouvement de rotation et celui des oscillations suivant la formule suivante :

$$
\tan (\theta / 2)=\frac{V_{\text {axiale }}}{V_{\text {rotation }}}
$$

Ce paramètre est très important pour les propriétés fonctionnelles de la surface. En effet, des études expérimentales ont montré que la consommation d'huile augmente avec l'angle de rodage [2,9]. Il est usuellement contrôlé à partir des images de Fax film en calculant une moyenne approximative de l'angle de rodage sur cinq échantillons.

La transformée de Radon permet, par ailleurs d'obtenir une valeur plus objective et plus précise de l'angle de rodage en le calculant sur l'ensemble de l'échantillon.

\subsubsection{Densité des stries $D_{\mathrm{s}}$}

La densité de stries, notée $D_{\mathrm{s}}$, est le nombre de stries par unité de surface (unité : stries. $\mathrm{mm}^{-2}$ ). Ce paramètre représente le rapport entre le nombre de stries (c'est-àdire le nombre des maxima locaux de la transformée de radon de la surface) et l'aire de la surface analysée.

Une fois que cette nouvelle méthodologie de caractérisation a été mise en place, les deux paragraphes suivants illustreront son application et son efficacité dans la définition des surfaces rodées. Une corrélation entre la texture de surface et les variables process ainsi que la capacité de la méthode à contrôler le process seront également démontrées. 
Tableau 1. Principaux paramètres appliqués du procédé de rodage.

\begin{tabular}{lc}
\hline \multicolumn{2}{c}{ Paramètres process } \\
\hline Vitesse axiale $\left(\mathrm{m} \cdot \mathrm{min}^{-1}\right)$ & 22 \\
Vitesse de rotation $\left(\mathrm{tr} \cdot \mathrm{min}^{-1}\right)$ & 190 \\
Type d'expansion & Mécanique \\
Vitesse d'expansion $\left(\mu \mathrm{m} \cdot \mathrm{s}^{-1}\right)$ & 1,5 \\
Nombre de pierres de rodage & 6 \\
Type de liant & Vitrifié \\
Type de grain & Carbure de silicium $(\mathrm{SiC})$ \\
Dimensions des pierres $(\mathrm{mm} \times \mathrm{mm} \times \mathrm{mm})$ & $6 \times 6 \times 70$ \\
\hline
\end{tabular}
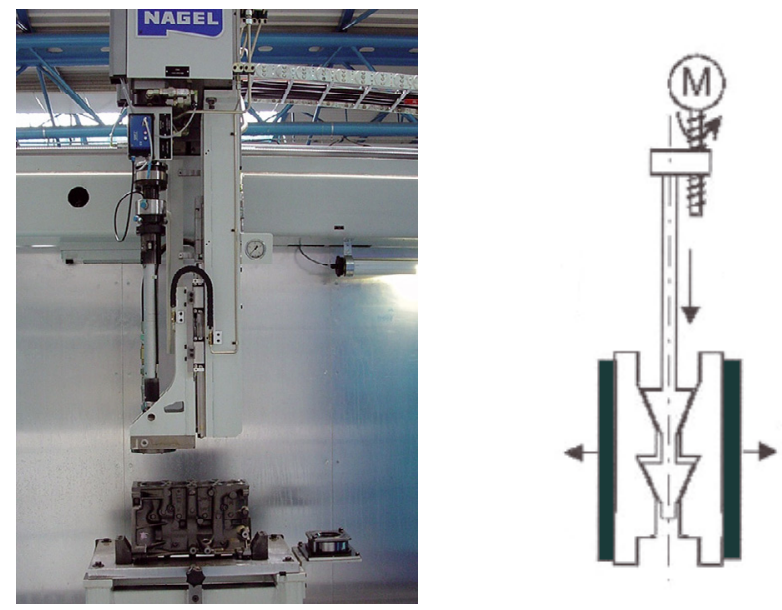

Fig. 7. Dispositif expérimental des tests de rodage.

\section{Influence des paramètres process sur la qualité du fini de surface}

Afin d'étudier l'impact des paramètres process sur la texture des surfaces rodées, des essais instrumentés ont été réalisés sur une machine de rodage mono-broche Nagel (cf. Fig. 7).

Des capteurs de puissance ont été placés au niveau du moteur de rotation afin de contrôler la puissance consommée pendant le rodage en relevant la puissance à vide et la puissance en charge du moteur. Les pièces usinées sont des carters cylindre en fonte grise à graphite lamellaire de moteur de voitures Renault quatre cylindres. Chaque cylindre a une longueur de 141,4 mm avec un diamètre moyen de 75,935 $\pm 0,0035 \mathrm{~mm}$. Les pièces sont initialement rodées à la phase d'ébauche et les différents tests porteront sur la phase de rodage finition. Pour chaque série de tests, les contrôles suivants ont été réalisés :

1. contrôle du diamètre à trois niveaux du fût pour les trois positions PMH, PM et PMB (Fig. 3) en utilisant un comparateur SUBITO ;

2. contrôle de la rugosité pour les trois niveaux PMH, $\mathrm{PM}$ et $\mathrm{PMB}$ en utilisant un profilomètre SURFASCAN sur une longueur de profil de $16 \mathrm{~mm}$ (Norme ISO 4287);
3. contrôle de l'aspect par la méthode du fax film décrite ci-dessus;

4. contrôle de la surface par une mesure 3D par un interféromètre WYKO NT 3300.

\subsection{Influence de la taille du grain abrasif}

Chacune des étapes de rodage engendre une empreinte spécifique sur le fini de la surface rodée. Une étude menée par El Mansori et al. a permis, par ailleurs, de ressortir l'action déterministe et fondamentale de l'étape de rodage finition dans le résultat final de la surface rodée [10]. Celleci définit la rugosité principale de la surface et corrige les irrégularités des étapes précédentes (alésage et rodage ébauche) sur toutes les échelles. Ainsi, on se propose de focaliser par la suite l'étude des effets de la granulométrie au cours de cette étape de rodage finition.

Afin d'étudier l'impact de la taille de grain abrasif à l'étape de finition, nous avons sélectionné différentes tailles de grains de carbure de silicium allant de $30 \mu \mathrm{m}$ à $380 \mu \mathrm{m}$. Le tableau suivant résume les différents paramètres process appliqués.

\subsubsection{Sur la rugosité de la surface}

À l'échelle de la rugosité, l'intervention du facteur granulométrie, illustrée par les paramètres fonctionnels de rugosité $(C r, C f$ et $C l)$, est critique en trois domaines caractéristiques (Fig. 8) :

- d'une part pour les tailles de grains inférieures à $60 \mu \mathrm{m}$, où règne un faible niveau de rugosité;

- d'autre part la zone de granulométrie comprise entre $60 \mu \mathrm{m}$ et $240 \mu \mathrm{m}$, où se trouvent les niveaux de rugosité intermédiaire et stable;

- enfin, la granulométrie supérieure à $240 \mu \mathrm{m}$, où la rugosité est maximale.

Outre l'identification de ces trois domaines caractéristiques, l'analyse multi-échelle de l'état de surface apporte une information importante sur l'évolution des échelles caractéristiques de la surface. En effet, les 

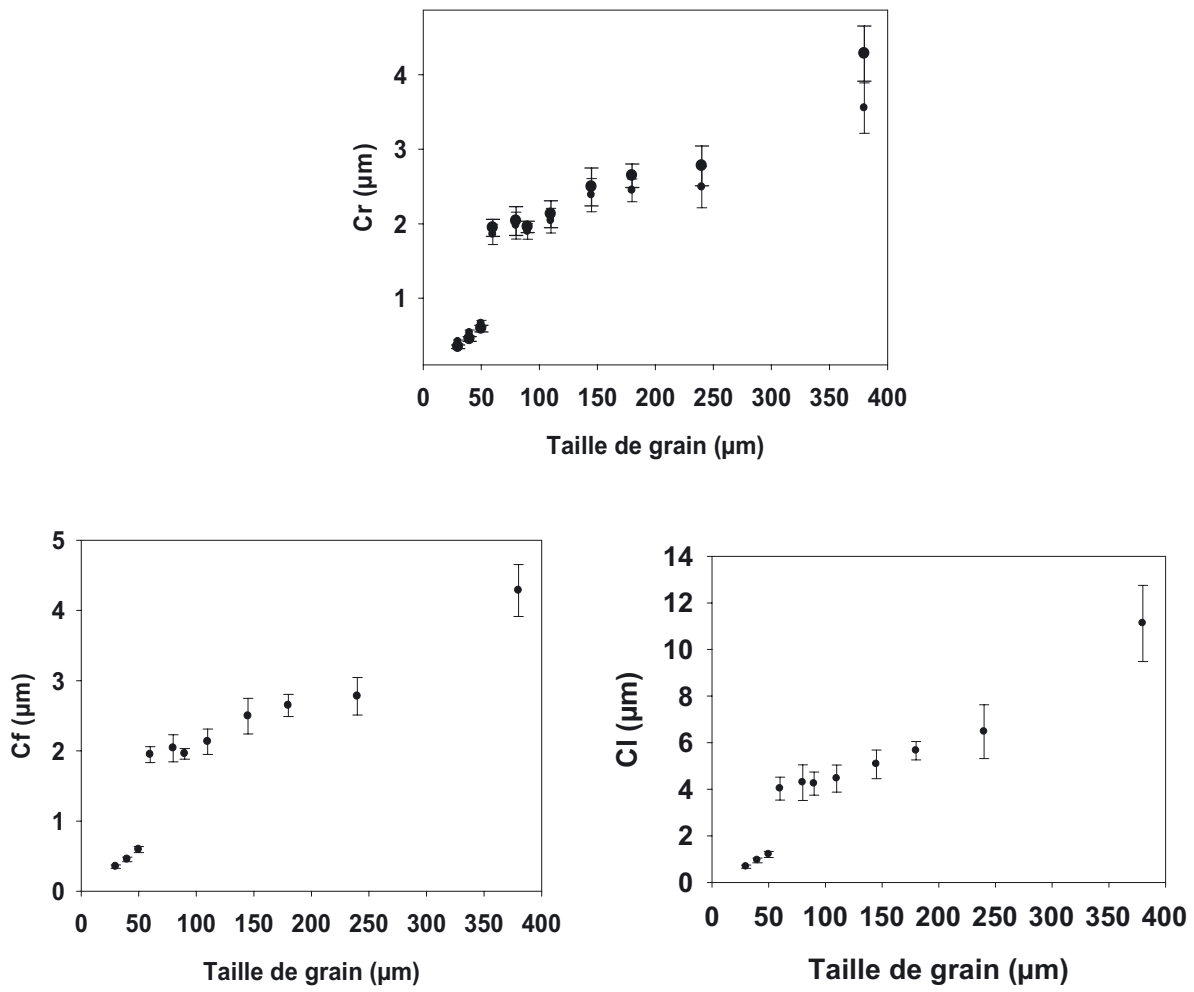

Fig. 8. Variations des paramètres fonctionnels de rugosité $C r, C f$ et $C l$ avec la taille des grains SiC. (Paramètres process : $V_{\exp }=4 \mu \mathrm{m} . \mathrm{s}^{-1}, V_{\mathrm{a}}=23 \mathrm{~m} \cdot \mathrm{min}^{-1}$ et $V_{\mathrm{r}}=190 \mathrm{tr} \cdot \mathrm{min}^{-1}$.)

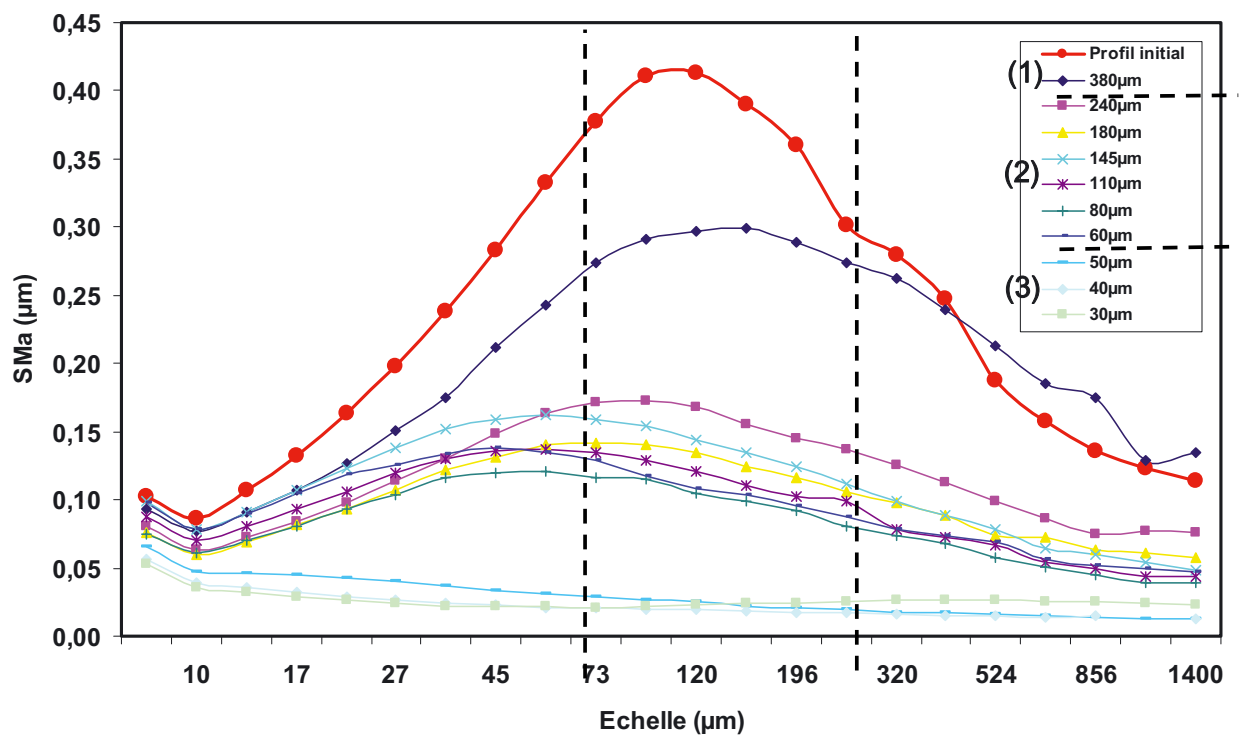

Fig. 9. Évolution des spectres multi-échelles de rugosité SMa fonction de la taille des grains.

spectres multi-échelles SMa des surfaces rodées par les différentes tailles de grains $\mathrm{SiC}$ montrent que (Fig. 9) :

- pour le domaine 1, les spectres de rugosité sont indépendants des échelles d'analyse. Une atténuation maximale de la rugosité est en effet observée sur toutes les échelles pour les tailles de grains de ce domaine $(<60 \mu \mathrm{m})$;
- pour le domaine 2, les spectres de rugosité dépendent de l'échelle d'analyse. Ils présentent une amplitude maximale à une échelle inférieure à celle du spectre de la surface initiale;

- pour le domaine 3, le spectre d'amplitude de rugosité ne présente qu'une simple atténuation de l'amplitude de rugosité. Aucune action sur l'échelle caractéristique de la surface n'est observée. 

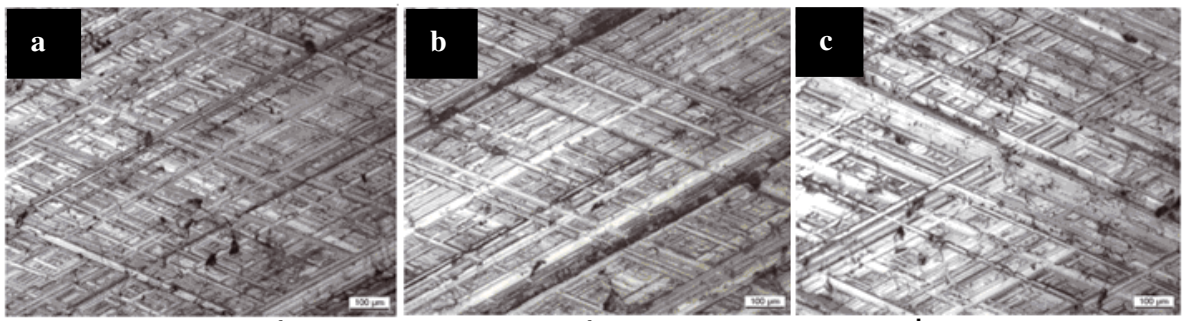

Fig. 10. Observation de l'aspect des surfaces rodées avec différentes de grains de SiC (a) $110 \mu \mathrm{m}$, (b) $180 \mu \mathrm{m}$ et (c) $240 \mu \mathrm{m}$.

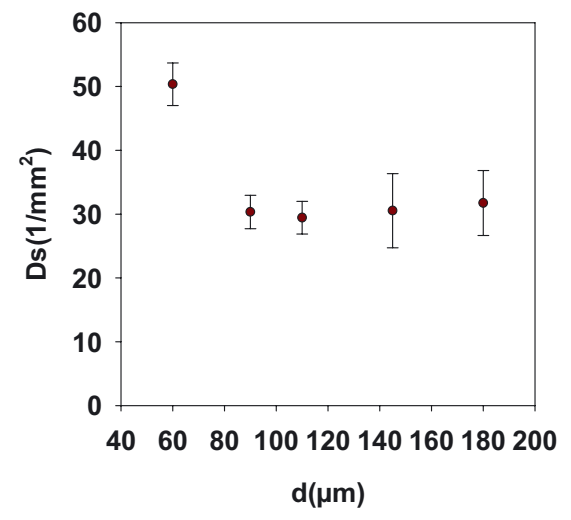

Fig. 11. Variations de la densité des stries $D_{\mathrm{s}}$ en fonction de la taille des grains $\mathrm{SiC}$.

\subsubsection{Sur l'aspect de surface}

L'observation au microscope optique des répliques des surfaces rodées issues de la méthode du fax film montre que l'augmentation des tailles de grains conduit à une striation large de la surface accompagnée d'une forte rugosité (Fig. 10).

Cette inspection visuelle est complétée par le calcul des densités de stries formées par les différentes tailles de grains. La figure 11 précise ce résultat et confirme que la densité des stries est élevée pour la taille de grains à $60 \mu \mathrm{m}$ alors qu'elle se stabilise pour les autres tailles de grains supérieures.

Ces variations de la qualité du fini de surface, par rodage, sous l'effet de la granulométrie sont le résultat des mécanismes physiques à l'origine de l'action des grains abrasifs. La mise en évidence de ces phénomènes est décrite dans le paragraphe ci-après.

\subsection{Identification des mécanismes physiques induits par le facteur de granulométrie}

Effectuons un calcul de l'énergie spécifique $E_{\mathrm{sp}}$ et du taux d'enlèvement de matière $Q_{w}$ relatifs à chaque cas de taille de grain et dans des conditions cinématiques de

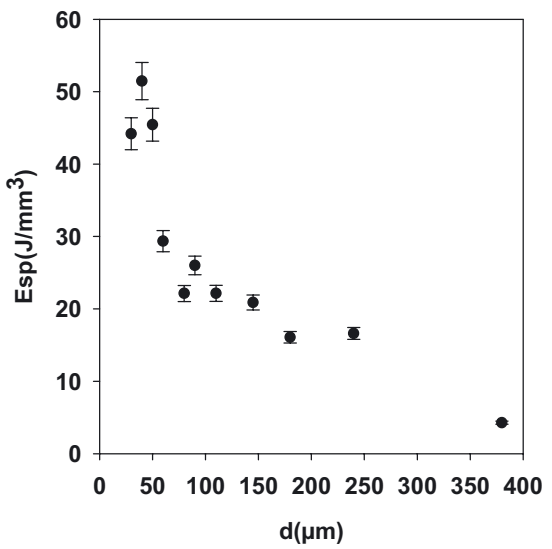

Fig. 12. Évolution de l'énergie spécifique avec la taille des grains $\mathrm{SiC}$.

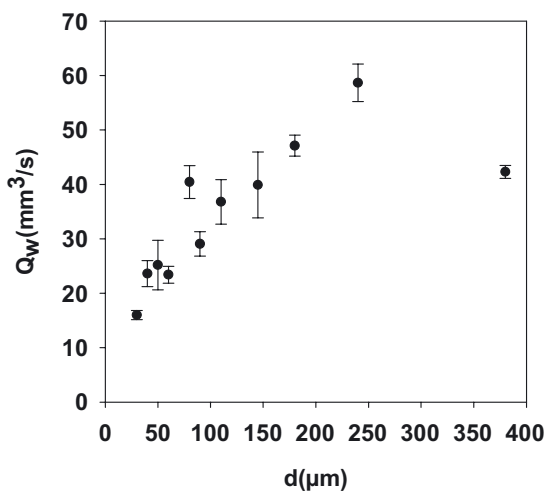

Fig. 13. Évolution du taux d'enlèvement de matière en fonction de la taille des grains abrasifs.

rodage identiques. Ces deux paramètres sont définis comme suit :

$$
\begin{aligned}
& E_{\mathrm{sp}}=\frac{P_{c} t}{W} \\
& Q_{w}=\frac{W}{t}
\end{aligned}
$$

où $P_{\mathrm{c}}$ est la puissance consommée, $t$ : le temps de rodage et $W$ le volume de matière enlevée. On constate que (Figs. 12 et 13) :

- les pierres de granulométrie inférieure à $60 \mu \mathrm{m}$ enregistrent une énergie de rodage très élevée avec un taux d'enlèvement de matière faible. Le processus de labourage par déformation plastique est ici privilégié. 

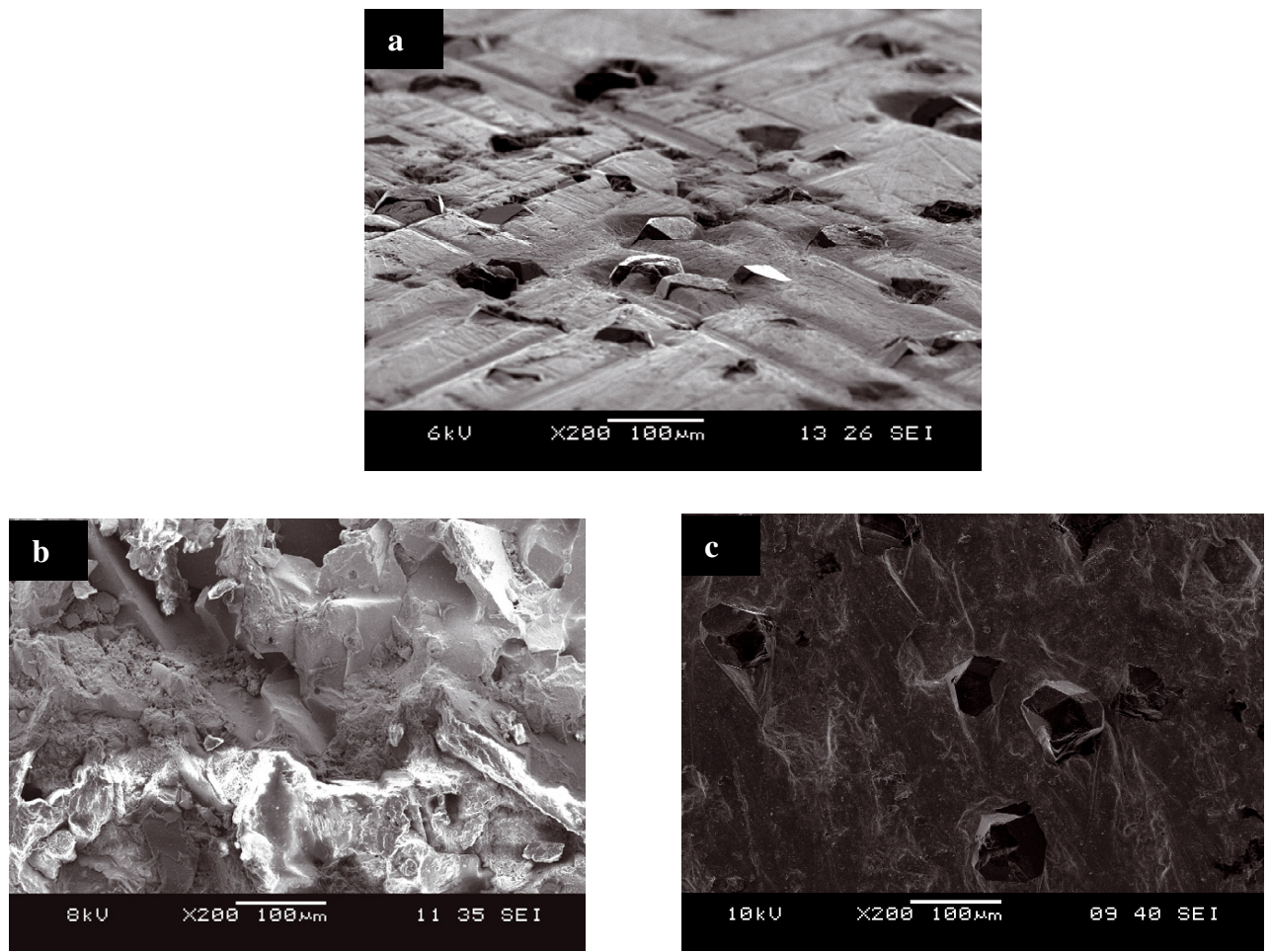

Fig. 14. Morphologie structurale des différentes pierres à base de diamant : (a) diamant métallique, (b) diamant vitrifié et (c) diamant résinoide.

La faible rugosité obtenue démontre que le mode d'action du rodage est dominé par déformation plastique des sommets et lissage des rugosités par frottement;

- l'énergie induite par le rodage entre les grains de taille supérieure à $60 \mu \mathrm{m}$ et inférieure à $240 \mu \mathrm{m}$ a été plus faible et plus stable. Ainsi, l'accroissement sensible de la rugosité issue du rodage résulte ici d'un couplage entre mécanismes de labourage et coupe;

- l'énergie atteinte pour une granulométrie supérieure à $240 \mu \mathrm{m}$ a été minimale. Le mécanisme de coupe est ici prépondérant et démontré par le taux de matière enlevé obtenu.

Ces résultats montrent d'une part la sensibilité de l'approche multi-échelle au paramètre process « grain abrasif $»$ et sa corrélation avec les mécanismes physiques qui régissent le procédé d'une autre part.

\subsection{Influence du type de liant abrasif}

On doit rappeler que le rodage diamant exploite des pierres abrasives agglomérées à base de grains diamants et à distribution aléatoire. La gamme de rodage diamant peut être déployée soit dans les trois étapes de rodage (ébauche, finition, plateau) avec des tailles de grains différentes; soit dans les phases de rodage ébauche et finition puis un passage à l'étape plateau avec du carbure de silicium vitrifié. Bien que cette gamme « diamant » améliore la productivité du process grâce aux performances contre l'usure des grains en diamant (dureté égale à 7000 contre 3000 pour le carbure de silicium à l'échelle de Knoop), l'utilisation des pierres en diamant métallique introduit des manteaux de tôle à cause du refoulement de la matière sur les bords des stries. Ils sont sous forme d'écailles de poisson qui frotteront mal avec les segments surtout quand le glissement prend les écailles «à rebrousse-poil ». Ce type de texture de surface risque de détériorer la fonctionnalité de la surface et d'augmenter l'usure du système segment/piston/fût (SPF). Face à cette problématique, on propose ici de développer de nouvelles solutions de pierres abrasives pour définir la qualité des surfaces rodées. Celle-ci sera jugée en utilisant l'approche multi-échelle développée. La figure 14 montre les caractéristiques structurelles des deux nouvelles pierres abrasives testées comparées à celles du diamant métallique. Les trois types de pierres ont les mêmes dimensions $(6 \times 6 \times 70 \mathrm{~mm})$ et ont subi une phase de mise au rond par 10 passages de cycles de rodage finition afin de les dresser et aviver les grains. En résumé, ces pierres sont définies comme suit :

1. diamant métallique (Dia-M) : composé d'un liant métallique en bronze et de grains en diamant de taille égale à $91 \mu \mathrm{m}$ et d'une concentration de $50 \%$ par rapport au volume total de la pierre. Le liant présente, dans ce cas, une structure fermée et marquée par les traces de striation générées lors du mouvement hélicoïdal des pierres (Fig. 14a);

2. diamant vitrifié (Dia-V) : composé d'un liant vitrifié (verre $\mathrm{C} 7$ ) et de grains diamant de taille moyenne égale à $107 \mu \mathrm{m}$ et de concentration égale à $50 \%$ du 

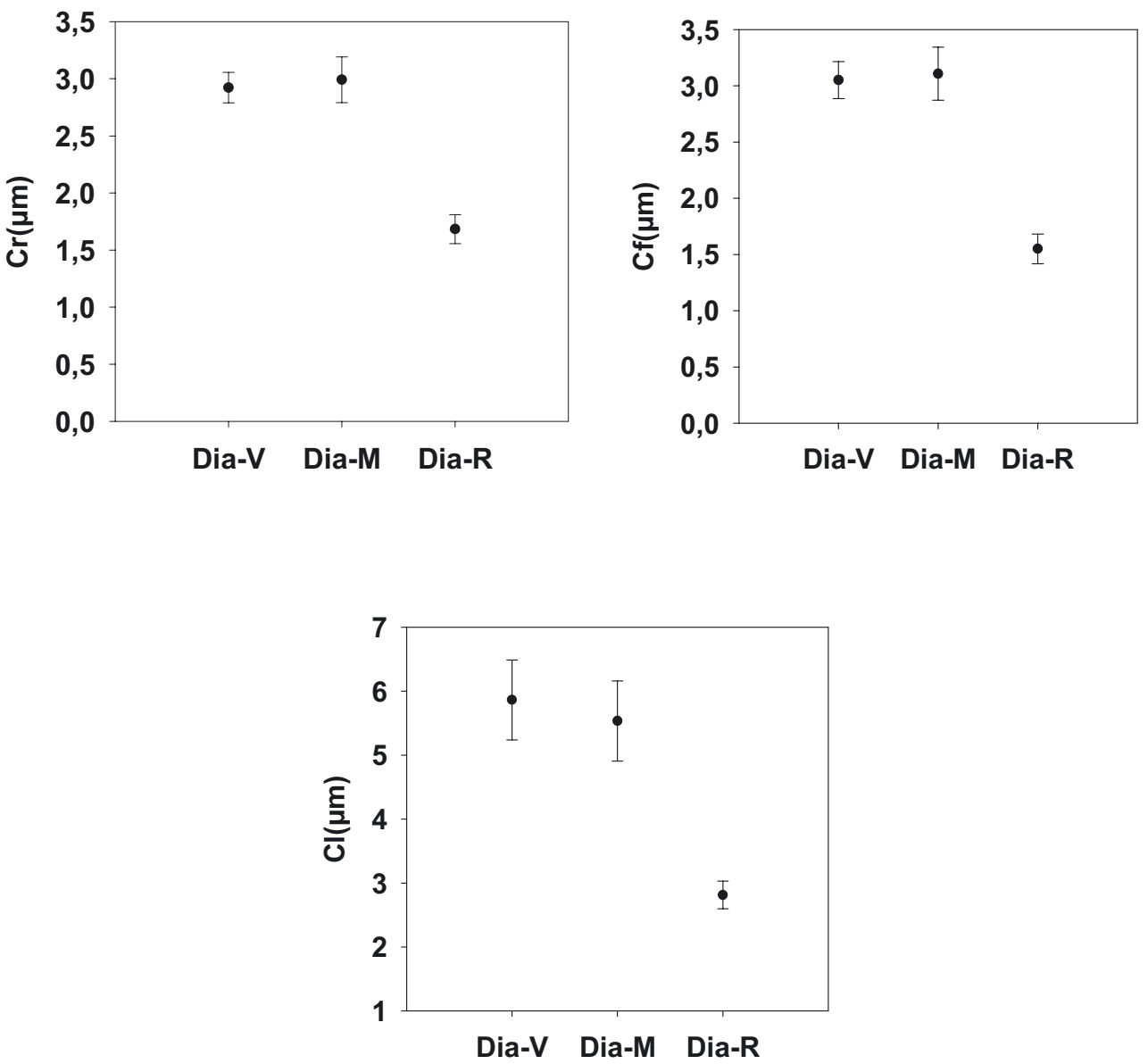

Fig. 15. Variations des paramètres d'état de surface en fonction du type de liant agglomérant les grains diamant.

volume total de la pierre. Des grains de carbure de silicium de taille égale à $60 \mu \mathrm{m}$ sont rajoutés pour assurer un meilleur accrochage des grains. La structure des pierres est plus ouverte et présente plus d'espace inter-granulaire par rapport au diamant métallique (Fig. 14b) ;

3. diamant résinoide (Dia-R) : composé d'un liant résinoide et de grains diamant de taille moyenne égale à $91 \mu \mathrm{m}$. Les grains abrasifs sont revêtus de nickel pour mieux les retenir dans la matrice. Cette pierre donne une structure moins ouverte que le diamant vitrifié mais contient tout de même des pores permettant de réduire les phénomènes d'encrassement des copeaux (Fig. 14c).

\subsubsection{Qualité du fini de surface produit}

Concernant les irrégularités de la surface en termes d'état de surface, le diamant vitrifié et le diamant métallique n'ont pas de différences entre les paramètres $C r, C f$ et $C$ alors que le diamant résinoide produit une surface plus fine (Fig. 15).

L'analyse multi-échelle permet une fois de plus d'apporter plus de précisions quantaux différentes variations

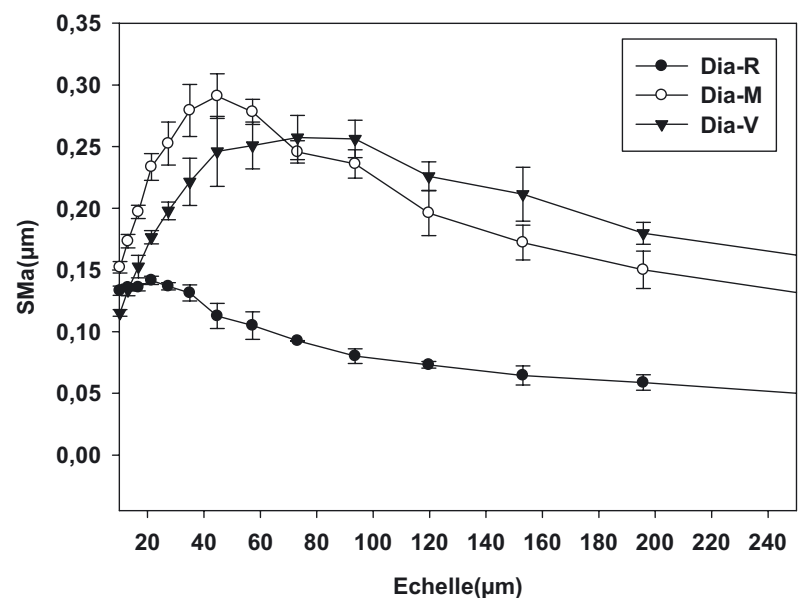

Fig. 16. Spectres multi-échelles de rugosité pour les pierres à grains diamants et à liants vitrifié, résinoide et métallique.

de la rugosité de la surface. La figure 16 montre l'effet d'échelle dû au changement de liant :

- pour les échelles supérieures à $100 \mu \mathrm{m}$, on remarque un simple décalage des spectres de rugosité, le Dia-R étant le plus fin ; 


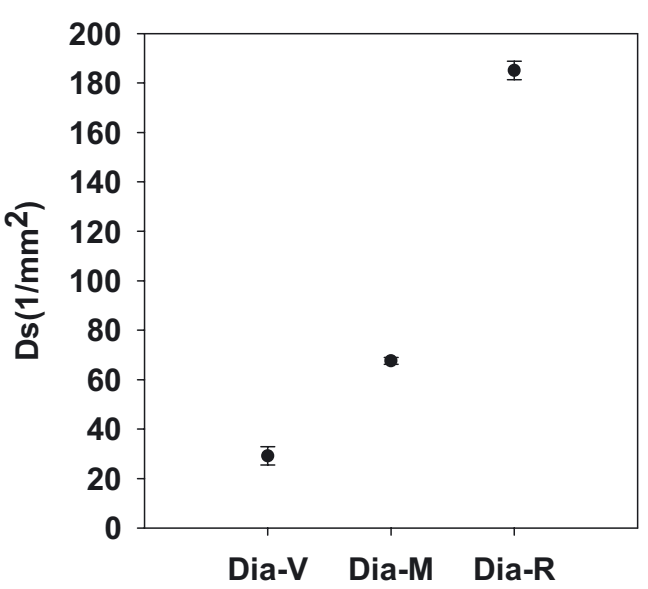

Fig. 17. Variation du paramètre $D_{\mathrm{s}}$ de densité des stries en fonction du type de liant.

- pour les échelles inférieures à $100 \mu \mathrm{m}$, différents comportements sont observés en fonction du liant :

- le diamant résinoide a une action de réduction maximale de la rugosité avec une légère augmentation pour les échelles inférieures à $30 \mu \mathrm{m}$;

- le diamant métallique et le diamant vitrifié présentent un maximum de rugosité pour les échelles respectives de $60 \mu \mathrm{m}$ et $80 \mu \mathrm{m}$.

Cette analyse montre que le liant impacte directement les échelles de finition de topographie de la surface.

Au niveau de l'aspect, le type du liant influe fortement la formation des stries. Comme illustré sur la figure 17, on observe une augmentation de la densité des stries pour le diamant résinoide alors qu'elle est minimale pour le diamant vitrifié.

\section{Contrôle des performances du process}

L'efficacité des outils multi-échelle a également été démontrée à travers une application de production relative à la détection d'un défaut de consommation d'huile élevée pour une machine de rodage nouvellement installée alors qu'aucune variable process n'a été changée par rapport à celles utilisées pour les machines déjà en fonction.

Nous avons, en effet, relevé des échantillons de surfaces rodées avec la machine défectueuse et avec une machine fonctionnelle et les avons contrôlés à l'interféromètre puis leur avons appliqué la méthode multi-échelle comme décrit précédemment.

En se référant aux résultats de la figure 18, on remarque que les deux machines ont des spectres de rugosité différents au niveau des petites échelles. Ce résultat est complété par l'observation d'une variation de la densité des stries issue des deux machines de rodage, comme le montre la figure 19. La densité de stries est en effet très liée à l'effort normal appliqué sur les pierres. Celuici définit les modes de régénération des pierres, et par la suite la qualité de la surface obtenue, puisqu'il est responsable du pouvoir d'indentation des grains dans les

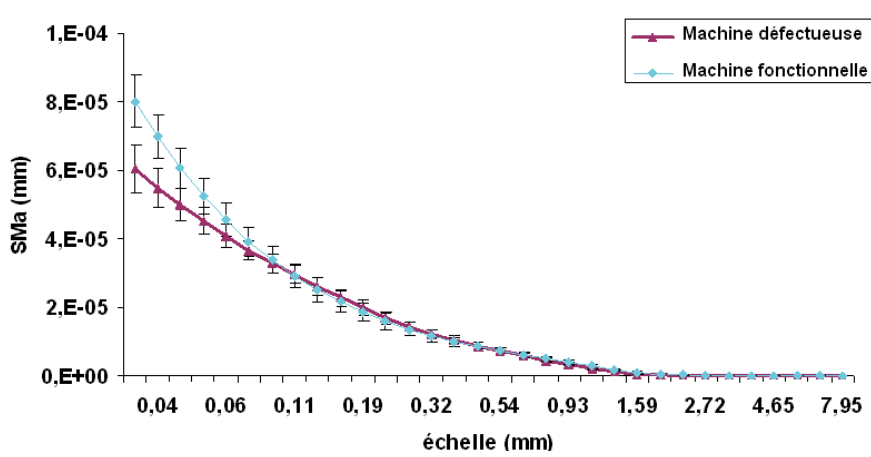

Fig. 18. Comparaison des spectres multi-échelles de rugosité entre les deux machines de rodage en appliquant les mêmes variables process.

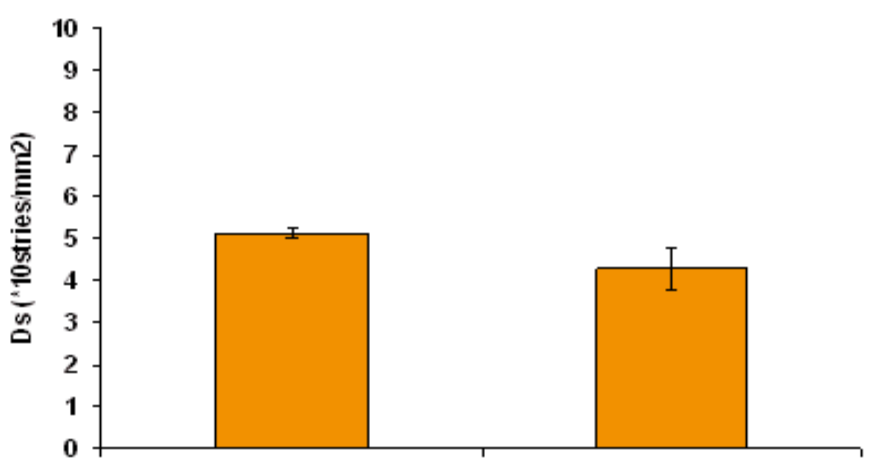

Machine fonctionnelle Machine défectueuse

Fig. 19. Variation de la densité de stries entre les deux machines de rodage en appliquant les mêmes variables process.

surfaces rodées. Ainsi, un contrôle direct des efforts normaux nécessaires à chacune des deux machines a montré un écart de l'effort normal appliqué sur les pierres de rodage. Il est, en effet, réduit pour la machine défectueuse à cause d'une défaillance au niveau du système mécanique de l'outil de rodage (jeu entre le cône et le porte-outil). Il est important de noter que les différentes méthodes classiques de contrôle d'état de surface n'ont pas permis de détecter ces fines variations entre les deux machines, ainsi que leurs rapports avec les variations de l'effort normal.

\section{Conclusions}

La caractérisation usuelle des surfaces rodées est basée sur une discrimination de la topographie par mesures profilométriques et de l'aspect par visualisation microscopique. La subjectivité de cette méthode et sa non-adéquation aux surfaces anisotropes conduisent à une perte d'informations et rendent difficile le contrôle et la maîtrise du procédé ainsi que la classification des surfaces obtenues.

De cette étude de qualité des surfaces obtenues après le procédé de rodage, il ressort que la décomposition multiéchelle est un outil puissant pour une caractérisation objective des finis de surfaces. Notre approche concerne l'exploitation de cette méthode multi-échelle, afin d'établir 
des relations directes entre les paramètres process et la texture de la surface obtenue. Nous avons mis en évidence l'apport de la méthodologie dans la compréhension et l'analyse de différentes configurations de rodage, notamment dans les cas de variations de la granulométrie des carbures de silicium, et la variation du liant dans le cas du rodage diamant. Les spectres multi-échelle offrent plus de visibilité sur les modifications de la surface et les variations des échelles activées en changeant les variables process. Ils montrent aussi une corrélation avec les mécanismes physiques activés pendant le process.

La définition du paramètre densité de stries revêt aussi d'une importance particulière. Il permet non seulement d'avoir une caractéristique objective et quantifiable de la surface mais montre aussi sa capacité à distinguer des surfaces rodées usinées avec des variables process différentes.

\section{Références}

[1] G. Monteil, C. Lebeaud, Réduction des frottements dans le contact segment-piston-chemise d'un moteur thermique, contribution à l'amélioration du rendement, dans Ingénieurs de l'Automobile (1998) pp. 46-51

[2] L. Shengfeng, K. Iyer, S.J. HU, Functional characterization of surface roughness generated by plateau honing process using wavelet analysis, Society of Automotive Engineers 113 (2004) 712-718

[3] J. Abbott, F.A. Firestone, Specifying surface quality, Mech. Eng. 55 (1933) 569-572
[4] M. Harvier, G. Chaumond, CNOMO, Fascicule de formation États de surface, GE40-082N

[5] E. Mainsah, J.A. Greenwood, D.G. Chetwynd, Metrology and properties of engineering surfaces, Kluwer Academic Publishers, 2001, pp. 243-276

[6] L. Sabri, S. Mezghani, M. El Mansori, J.V. Le-Lan, Toward an objective 3D description for quality assessment of manufactured honed surfaces, NAMRC 37 conference, May 20-22, Greenville, SC, USA, 2009

[7] S. Mezghani, M. El Mansori, A. Massaq, P. Ghidossia, Correlation between surface topography and tribological mechanisms of the belt-finishing process using multiscale finishing process signature, C. R. Mecanique 336 (2008) 794-799

[8] F. Argüeso, J. Gonzalez-Nuevo, J.L. Sanz, L. Toffolatti, P. Vielva, D. Herranz, M. Lopez-Caniego, The mexican hat wavelet family: application to point source detection in cosmic microwave background maps, proceedings of the 13th European Signal Processing Conference, Antalya, Sept 4-8, 2005

[9] D.K. Srivastava, A.K. Agarwal, J. Kumar, Effect of liner surface properties on wear and friction in a non-firing engine simulator, Mat. Design 28 (2007) 1632-1640

[10] M. El Mansori, S. Mezghani, L. Sabri, H. Zahouani, On the concept of process signature in the analysis of multistage surface formation, Surf. Eng. 26 (2010) 216-223

[11] L. Sabri, M. El Mansori, Process variability in honing of cylinder liner with vitrified bonded diamond tools, Surf. Coat. Technol. 204 (2009) 1046-1050 